\title{
Correction to: Accompaniment of Time-Lapse Parameters and Cumulus Cell RNA-Sequencing in Embryo Evaluation
}

\author{
Azam Govahi ${ }^{1,2}$. Fatemehsadat Amjadi ${ }^{1}$ Mohammad-Hossein Nasr-Esfahani ${ }^{3} \cdot$ Ehsan Raoufi ${ }^{4}$. \\ Mehdi Mehdizadeh ${ }^{5}$ (D)
}

Published online: 6 December 2021

(c) Society for Reproductive Investigation 2021

\section{Correction to: Reprod Sci}

https://doi.org/10.1007/s43032-021-00748-3

This article was updated to correct the affiliation of author Mehdi Mehdizadeh and to correct the Iran National Science Foundation grant number in the Funding section.

Publisher's note Springer Nature remains neutral with regard to jurisdictional claims in published maps and institutional affiliations.

The original article can be found online at https://doi.org/10.1007/ s43032-021-00748-3.

Mehdi Mehdizadeh

mehdizadeh.m@iums.ac.ir

1 Department of Anatomy, School of Medicine, Iran University of Medical Sciences, Tehran, Iran

2 Student Research Committee, Iran University of Medical Sciences, Tehran, Iran

3 Department of Animal Biotechnology, Reproductive Biomedicine Research Center, Royan Institute for Biotechnology, ACECR, Isfahan, Iran

4 Department of Medical Biotechnology, School of Allied Medical Science, Iran University of Medical Sciences, Tehran, Iran

5 Reproductive Sciences and Technology Research Center, Iran University of Medical Sciences, Tehran, Iran 